\title{
MEMS FLOW SENSORS FOR NANO-FLUIDIC APPLICATIONS
}

\author{
S. Wu, Q. Lin, Y. Yuen and Y.C. Tai \\ Caltech Micromachining Laboratory \\ Electrical Engineering, 136-93 \\ California Institute of Technology \\ Pasadena, CA 91125, USA
}

\begin{abstract}
This paper presents micromachined thermal sensors for measuring liquid flow rates in the nanoliter-per-minute range. The sensors use a boron-doped polysilicon thinfilm heater that is embedded in the silicon nitride wall of a microchannel. The boron doping is chosen to increase the heater's temperature coefficient of resistance within tolerable noise limits, and the microchannel is suspended from the substrate to improve thermal isolation. The sensors have demonstrated a flow rate resolution below $10 \mathrm{~nL} / \mathrm{min}$, as well as the capability for detecting micro bubbles in the liquid. Heat transfer simulation has also been performed to explain the sensor operation and yielded good agreement with experimental data.
\end{abstract}

\section{INTRODUCTION}

The emerging technology of Micro Electro Mechanical Systems (MEMS) is enabling micro fluidic systems for many applications, such as micro chromatography, biochemical detection, and mass spectrometry. While precise flow control is generally needed in these applications, existing commercial sensors are inadequate for this purpose because of their limited sensitivity, large size, high dead volume, and difficulties in interfacing with microfluidic devices.

To address the need for precise flow control in microfluidic systems, MEMS technology has been used to develop micro sensors for liquid flow rate measurement [1-4]. Achieving a measuring resolution of up to a few microliters per minute, both thermal [1-3] and non-thermal [4] transducing principles have been used, and channels built using chip-bonding techniques have been employed as the flow-carrying device [1-2].

This paper presents a class of MEMS flow sensors that demonstrate the first capability of measuring liquid flow rates below $10 \mathrm{~nL} / \mathrm{min}$, as well as detecting passage of micro bubbles in the flow. These thermal sensors use a boron-doped polysilicon thin-film resistor embedded within the wall of a microchannel. The microchannel, a key feature for our sensors, is fabricated using surface micromachining technology [5] (without chip-bonding), and is released from the silicon substrate using $\mathrm{BrF}_{3}$ dry etching [6] for improved thermal isolation. The doping concentration for the polysilicon resistor is optimally chosen for an increased temperature coefficient of resistance, while keeping the noise level within tolerable limits. The subsequent sections describe the design, fabrication, testing, as well as heat transfer analysis of our flow sensors.

\section{DESIGN}

The basic operating principle of the flow sensor utilizes the micro convective heat transfer from a heated polysilicon thin-film sensing element (hereafter referred to as a heater) to a liquid flowing inside a microchannel. Figure 1 illustrates such a MEMS microchannel flow sensor approach. The polysilicon heater is built on the channel wall. By measuring the sensor temperature, the liquid flow rate can be calculated.

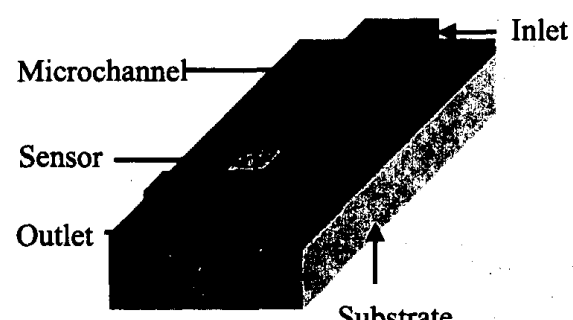

Figure 1. A microchannel-based flow sensing approach.

Since the channel is in direct contact with the substrate, the heat loss from the heater to the substrate may considerably reduce the sensor's sensitivity to the fluid flow. Figure 2 shows an improved approach, in which a portion of the substrate lying below the channel is removed to achieve better thermal isolation. To minimize the effect of channel wall deflection, the heater is located close to the channel outlet. 


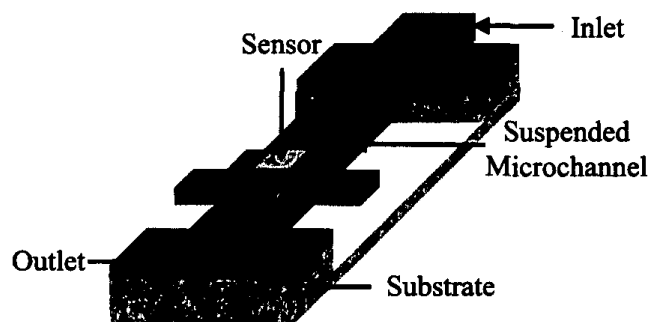

Figure 2. An improved flow sensing approach with a suspended microchannel.

Since the sensitivity of the sensor also depends on the heater's temperature coefficient of resistance (TCR), we investigated three sensor designs shown in Figure 3(ac). The microchannels in all three designs have typical dimensions of $2000 \times 20 \times 2 \mu \mathrm{m}^{3}$. In the first design (Figure 3(a)), the microchannel lies directly on the substrate, and the polysilicon heater is heavily doped with boron (concentration $2 \times 10^{19} \mathrm{~cm}^{-3}$ ). In comparison, the second design as shown in Figure 3(b), the doping concentration of the polysilicon thin film is reduced to $2 \times 10^{18} \mathrm{~cm}^{-3}$. This increases the heater's TCR (while keeping the noise level within tolerable limits), and hence sensitivity to the flow. Finally, the third design, depicted in Figure 3(c), employs a suspended microchannel, which results in improved thermal isolation. Compared with Figure 3(b), the lightly doped heater length is extended to the channel's entire transverse dimension. This increases the heater's resistance and hence reduces inaccuracies arising from the resistance of the leads and wires. To summarize, these three designs allow us to investigate the effects of channel suspension and heater temperature sensitivity.

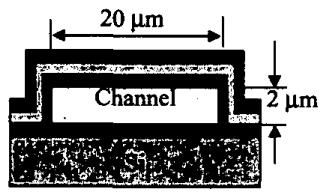

(a) Sensor \#1

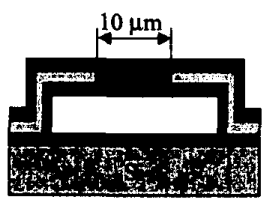

(b) Sensor \#2

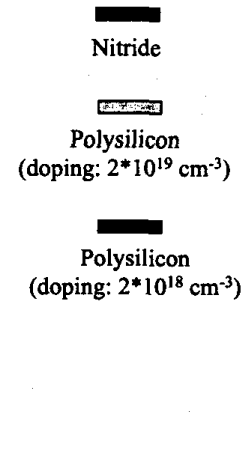

\section{FABRICATION}

The fabrication process of the flow sensor, including the microchannel and integrated heating element (Figure 4), involves the following steps. (1) To start, a $1 \mu \mathrm{m}$-thick silicon nitride layer is deposited onto a bare silicon wafer. (2) A $2 \mu \mathrm{m}$ PSG layer is then deposited on top of silicon nitride. (3) The PSG layer is patterned to define the microchannel geometry. (4) Anther $0.6 \mu \mathrm{m}$ layer silicon nitride is deposited as the top wall of the microchannel. (5) The PSG is etched away by HF to form the microchannel. (6) $0.5 \mu \mathrm{m}$ of polycrystalline silicon is deposited as the sensor material. (7) Polysilicon is doped by $\mathrm{B}^{+}$ion implantation, and followed by annealing at $1000^{\circ} \mathrm{C}$ for 30 minutes. (8) Polysilicon is patterned by plasma dry etching to define sensor geometry. (9) The sensors are sealed by uniformly depositing $0.6 \mu \mathrm{m}$ of silicon nitride. Then the inlet and outlet are opened by KOH. (10) Contact opening and $\mathrm{Al}$ metalization form connections between the macro world and the sensing element. (11) Finally, thermal isolation of the channel is achieved by $\mathrm{BrF}_{3}$ isotropic bulk etching of the silicon underneath the channel.

Figure 5 shows a fabricated flow sensor with two polysilicon elements integrated to a micro channel setting on silicon substrate.

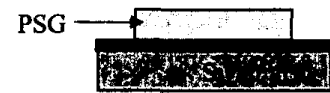

1. LPCVD nitride deposition $(1 \mu \mathrm{m})$

2. LPCVD PSG deposition $(\sim 2 \mu \mathrm{m})$

3. Patterning PSG

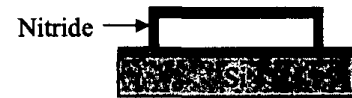

4. LPCVD nitride deposition $(0.5 \mu \mathrm{m})$

5. Etching PSG sacrificial layer

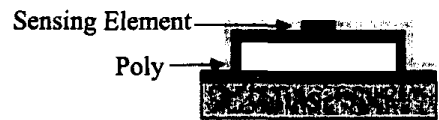

6. LPCVD polysilicon deposition $(0.5 \mu \mathrm{m})$

7. $\mathrm{B}^{+}$implantation

8. Patteming polysilicon sensors

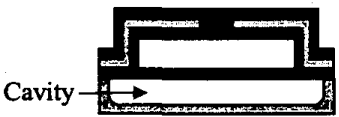

9. LPCVD polysilicon deposition $(0.6 \mu \mathrm{m})$

10. Contact hole opening and metallization

11. Si undercutting below sensor area using $\mathrm{BrF}_{3}$

Figure 4. Process flow of the microchannel flow sensor with a sensing element embedded in the channel wall.

Figure 3. Three flow sensor designs. 


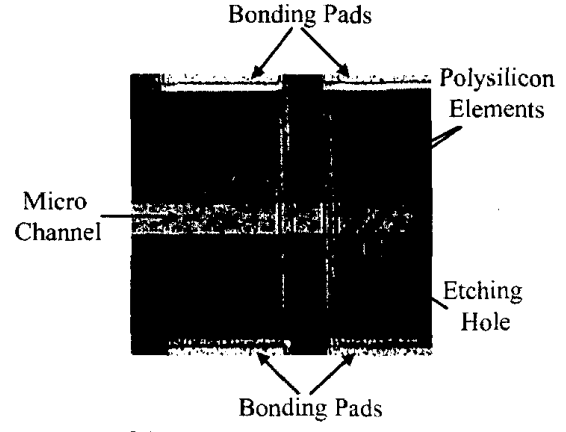

Figure 5. A fabricated flow sensor with polysilicon resistors and a micro channel on the silicon substrate.

\section{EXPERIMENTAL SETUP}

The flow measurements were conducted with three different types of sensors (Figure 3 ) using the apparatus illustrated in Figure 6. DI water was forced into the microchannel by pressurized microelectronic grade nitrogen gas from a reservoir tank. The volumetric flow rate was then measured by timing the distance traveled by the water meniscus past marked gradations on a $10 \mu \mathrm{L}$ glass pipette. Since we can estimate the tolerances on each of the dimensions and the timer, we can also estimate the associated error in the flow rate measurement. The relative errors were typically within $4 \%$. The resulting flow data was used as a standard for calibrating the sensors. (We refer to this data as the flow rate standard.) Data for each set of flow parameters (at various inlet and outlet pressures) was taken in a time period between thirty minutes and twenty-four hours, depending on the flow rate. Each flow rate was obtained by averaging over several measurements for each inlet pressure.

The sensor output was taken simultaneously with the flow measurements to give a correlation between sensor readings and flow rate. The sensor was tested and calibrated in an isothermal chamber with $\pm 0.05^{\circ} \mathrm{C}$ temperature control to minimize ambient temperature fluctuations. The sensors were calibrated against an Omega DP251 Precision Temperature Meter using an RTD probe. With the sensors biased between $70 \mu \mathrm{A}$ to $100 \mu \mathrm{A}$, the voltage readings were taken using an HP $34970 \mathrm{~A}$ data acquisition system, thus enabling us to track the time history of sensor readings. The temperature calibration combined with sensor measurements provided thermal data essential for comparison to finite element simulation. Unlike the flow measurements, the sensor measurements were much faster $(\sim 10-60$ samples/s) and 100 data points were taken per flow rate measurement.

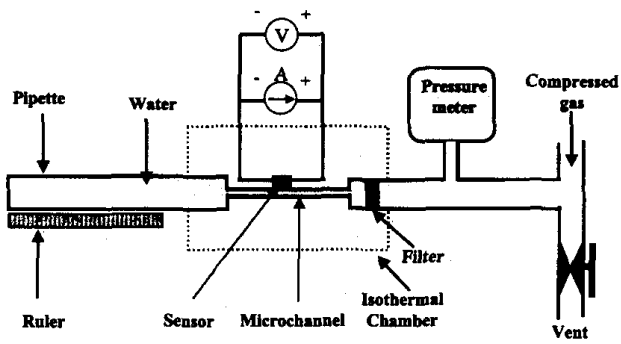

Figure 6. Schematic of the calibration system for MEMS flow sensors.

\section{TEST RESULTS AND DISCUSSION}

We discuss the calibration of the heater resistance as a function of the temperature, the voltage output in response to fluid flow, and flow-induced heater temperature change. Figure 7 shows the temperature dependence of sensor resistance for the three sensors. In the temperature calibration range the resistances decrease linearly with temperature, and thus can be characterized as follows.

$$
\frac{R(T)}{R\left(T_{0}\right)}=1+\alpha\left(T-T_{0}\right)
$$

where $\alpha$ is the temperature coefficient of resistance (including effects of lead and wire resistance change). For sensors $\# 1, \# 2$ and $\# 3, \alpha$ is found to be $-0.17 \% /{ }^{\circ} \mathrm{C}$, $-0.56 \% /{ }^{\circ} \mathrm{C}$ and $-0.67 \% /{ }^{\circ} \mathrm{C}$, respectively. Sensors $\# 2$ and \#3 have a higher TCR than \#l because of lower doping of the heating region [5]. Compared to sensor \#2, sensor \#3 has a larger length with lower doping concentration, and hence a slightly higher TCR. According to our calibration, the temperature resolution of all three sensors is better than $0.05^{\circ} \mathrm{C}$.

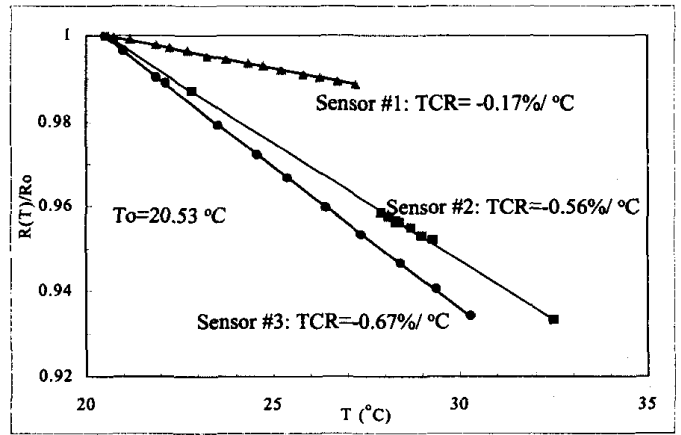

Figure 7. Temperature dependence of three types of sensors. 
The sensitivity of a sensor, denoted $S_{V}$, is defined as the ratio between the changes in output voltage and flow rate, i.e.,

$$
\Delta V=S_{V} \Delta \mathrm{Q}
$$

While $S_{V}$ varies for different flow rates, the sensitivity can be assessed in an average sense by the slope of a straight line fitted to the measurement data. Figure 8 shows the measured sensor response to the volumetric flow rate of DI water in the channel under constant current mode, and corresponding fitting lines. We can see that under the same constant current bias at $100 \mu \mathrm{A}$, sensor \#2 is much more sensitive, Furthermore, even at $70 \mu \mathrm{A}$ bias current, sensor $\# 3$ is 10 times more sensitive to the flow than sensor \#2.

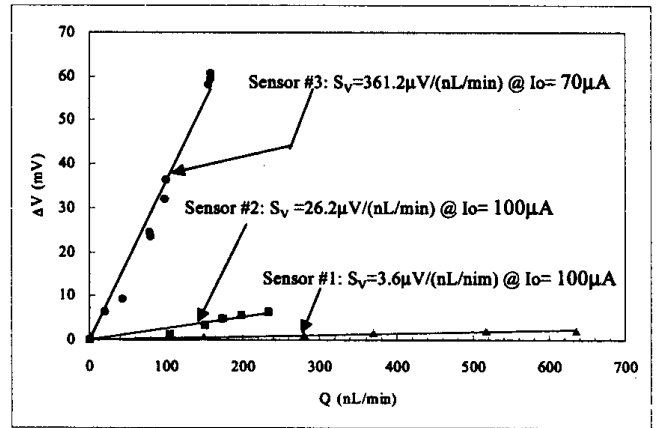

Figure 8. Characteristics of the flow sensors with three different designs.

Electrically, the output voltage change can be written as

$$
\Delta V=I_{0}\left[R(T)-R\left(T_{0}\right)\right]=V_{0} \alpha \Delta T,
$$

where $V_{0}=I_{0} R\left(T_{0}\right)$ and $\Delta T=T-T_{0}$.

From (2) and (3), we have

$$
S_{V}=V_{0} \alpha \frac{\Delta T}{\Delta Q}=V_{0} \alpha S_{T}
$$

where $S_{T} \equiv \frac{\Delta T}{\Delta Q}$, called temperature-to-flow ratio, is related to the structure design and fluid properties.

Although $S_{V}$ is an important sensor characteristic, it involves the original bias voltage that is not related to the sensor design. Thus, it is more appropriate to normalize the sensitivity by the initial bias signal and define the relative sensitivity:

$$
S_{V / V_{0}}=\frac{\Delta V / V_{0}}{\Delta Q}=\alpha \frac{\Delta T}{\Delta Q}=\alpha S_{T}
$$

From equation (5), the relative sensitivity is proportional to both the TCR and $S_{T}$. Note that the former is related to the sensing material properties and the latter is related to the structure design.

Figure 9 shows the sensor temperature change versus flow rate. We see that for the same given power $(140 \mu \mathrm{W})$, the suspended flow sensor gives the best temperature-to-flow ratio $\left(0.026^{\circ} \mathrm{C} / \mathrm{nL} / \mathrm{min}\right)$, about 5 times better than the first two sensors. This indicates that better thermal isolation offered by channel suspension achieves better sensitivity.

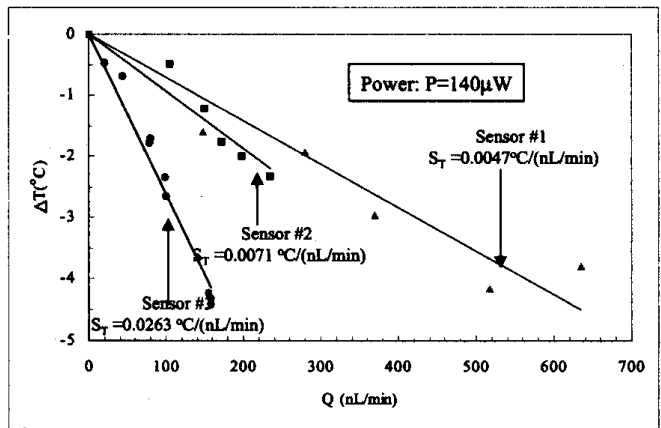

Figure 9. Temperature change of the sensors due to liquid flow.

Table 1 lists the TCR's, temperature-to-flow ratios and relative sensitivities of the three sensors for an input power of $140 \mu \mathrm{W}$. Sensor \#3 has the best sensitivity because of its large TCR and temperature-to-flow ratio.

\begin{tabular}{|c|c|c|c|}
\hline Sensor & $\# 1$ & $\# 2$ & $\# 3$ \\
\hline$\alpha\left(\% /{ }^{\circ} \mathrm{C}\right)$ & -0.17 & -0.56 & -0.67 \\
\hline$S_{7}\left({ }^{\circ} \mathrm{C} / \mathrm{nL} / \mathrm{min}\right)$ & 0.0047 & 0.0071 & 0.0263 \\
\hline$S_{V / N o}(\mathrm{ppm} / \mathrm{nL} / \mathrm{min})$ & 8 & 40 & 180 \\
\hline
\end{tabular}

Table 1. TCR's, temperature-to-flow ratios and relative sensitivities (input power: $140 \mu \mathrm{W}$ ).

\section{ERROR AND RESOLUTION ANALYSIS}

The resolution of the flow sensor is calculated by finding the maximum error associated with the sensor measurements and propagating the error through curve fitting, which gives the flow rate as a function of sensor voltage. It was found that the distribution of voltages for a particular flow rate can be modeled as a twoparameter Guassian distribution. Shown in Figure 10 is a typical distribution of sensor readings and the fitted Guassian distribution. About $70 \%$ of the readings lie 
within one standard deviation from the mean and $97 \%$ within two standard deviations. Thus, two standard deviations were taken as an adequate measure for the maximum error associated with an individual measurement. Further, according to the Central Limit Theorem [7], this error can be reduced by $\sqrt{N}$ through repeated measurements, where $\mathrm{N}$ is the number of samples.

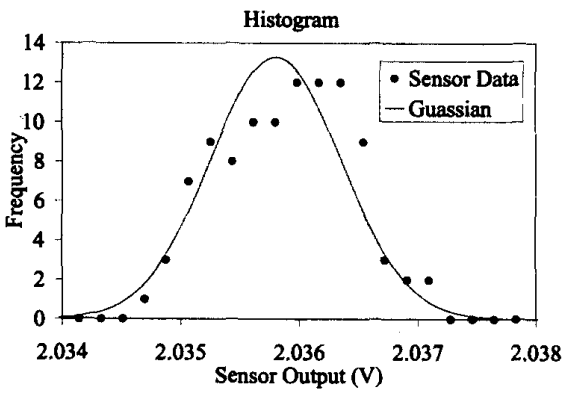

Figure 10. Histogram of a typical set of sensor measurements.

Once the errors for each measurement are known, the corresponding error in estimating the flow rate from the calibration can be calculated. For a typical single measurement, $\sigma_{\mathrm{V}}$ is about $500 \mu \mathrm{V}$ and thus corresponds to an error in flow rate measurement of about $10 \mathrm{~nL} / \mathrm{min}$. In this case, since the sensor system can resolve voltages to about $50 \mu \mathrm{V}$ after repeated measurements (100 times), the flow resolution is about $1 \mathrm{~nL} / \mathrm{min}$.

This resolution is then tested against actual measurements of flow rate using the sensor calibration and comparing it to the flow rate calibration standard. The two measurements agree well. For example, the flow rate standard gives $20.0 \pm 0.3 \mathrm{~nL} / \mathrm{min}$ at an inlet pressure of $27 \mathrm{psi}$, and the sensor calibration gives $21 \pm 1$ $\mathrm{nL} / \mathrm{min}$. Their absolute difference is smaller than the quadrate sum of their errors $|20.0-21|=1<$ $\sqrt{\left(0.3^{2}+1^{2}\right)}=1.2$. This shows that they are indeed the same measured quantity. The only difference is that the flow sensor can give a much quicker result.

\section{SENSOR MODELING}

To better understand the operation of the flow sensors, the heat transfer occurring in the sensors has been investigated. As a current is passed to the polysilicon heater, heat conduction occurs within the channel's solid structure, including the nitride walls, the embedded polysilicon heater, as well as the surrounding air. This heat conduction is coupled with forced convection heat transfer in the fluid flow. Assuming a laminar incompressible flow, the flow's velocity profile was found analytically [8]. Then, we employed the ABAQUS finite element package to solve the coupled conduction-convection problem, yielding the temperature distribution in both the channel and the flow. The average temperature $T$ for a given flow rate $Q$ was then computed.

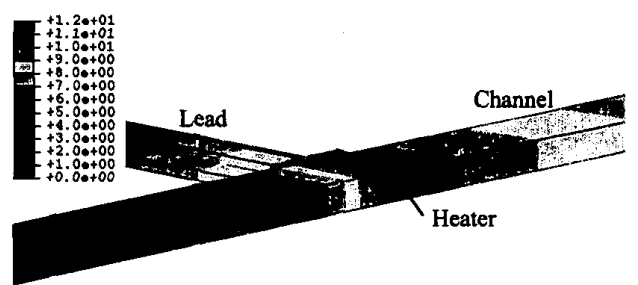

Figure 11. Temperature distribution along the channel (sensor \#3 at a flow rate of $100 \mathrm{~nL} / \mathrm{min}$ ).

Figure 11 shows the temperature distribution in the flow sensor (sensor \#3) at a flow rate of $100 \mathrm{~nL} / \mathrm{min}$. It can be seen from this figure that the size of the heated region is comparable along all the three spatial dimensions. In contrast to typical macroscale forced convection processes, this implies that heat conduction, in both the channel structure and the flow, in all three spatial directions is equally important for determining the heater's temperature.

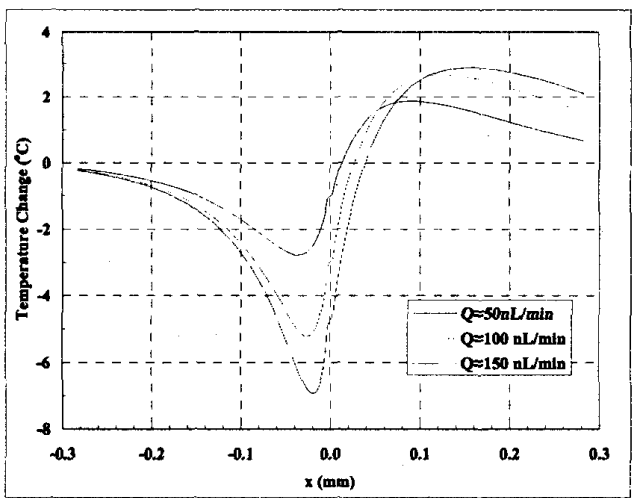

Figure 12. Distribution of the flow-induced temperature change along the channel for sensor \#3.

Since the heater temperature is a function of flow rate, i.e., $T=T(Q)$, the flow rate is reflected in the flowinduced temperature change $\Delta T=T(Q)-T(0)$. Figure 12 displays the distribution of $\Delta T$ along the channel for sensor \#3 at three different flow rates. In the figure the $x$-coordinate is measured along the channel with the heater at the origin. It is very interesting to observe that $\Delta T$ generally achieves its maximum away from the heater. Thus, the sensitivity of the flow sensor would be considerably improved if the flow rate is inferred from a separate temperature sensor located at an optimal distance away from the heater. Also, since the 
temperature change takes on opposite signs upstream and downstream of the heater, use of both upstream and downstream temperature sensors will allow the detection of flow direction.

The flow-induced sensor temperature change as a function of flow rate is plotted in Figure 13 for sensor \#3 along with the experimental data. From this figure it can be observed that the agreement between theory and experiment is quite good. The discrepancies may be due to uncertainties in the channel geometry and in the material properties.

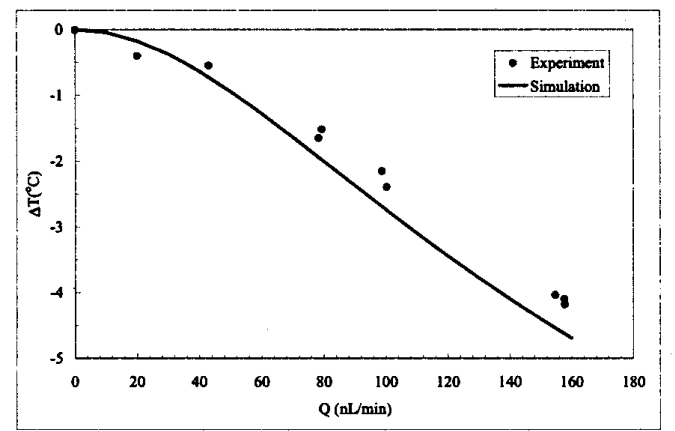

Figure 13. Simulated vs. measured flow-induced temperature change for sensor \#3.

\section{BUBBLE DETECTION}

Finally, our flow sensors can not only measure flow rates, but also detect the presence of air bubbles. In Figure 6 the passage of air bubbles is logged using realtime data acquisition. Since air has a lower heat capacity and conductivity than water, the sensor sees a sudden temperature rise, shown as spikes in Figure 15. Further investigation is underway to investigate the possibility of detecting foreign particles other than air bubbles. This can be particularly useful in microchemical reaction applications where detection of bubbles or particles is important.

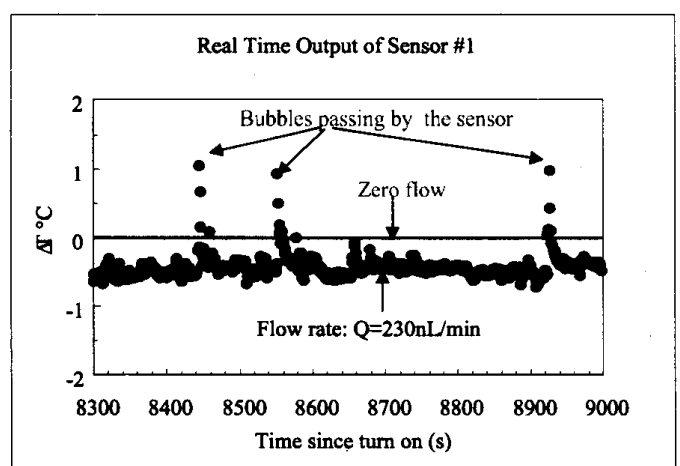

Figure 14. Real time output of sensor \#1. Temperature spikes indicate passage of an air bubble.

\section{CONCLUSION}

We have successfully developed liquid flow sensors that are integrated into a microchannel. The sensor sensitivity can be increased by switching from heavy doping $\left(2 \times 10^{19} \mathrm{~cm}^{-3}\right)$ to relatively light doping $\left(2 \times 10^{18}\right.$ $\mathrm{cm}^{-3}$ ), as well as removing a portion of silicon substrate underneath the sensor for better thermal isolation. The flow sensor has demonstrated a resolution of $10 \mathrm{~nL} / \mathrm{min}$ for single measurement and $1 \mathrm{~nL} / \mathrm{min}$ for averaged multiple measurements. In addition, heat transfer simulation was performed to study the operating principle of the flow sensors, and was found to agree with experimental data. Finally, it was demonstrated that micro bubbles in the liquid flow are detectable by the real time recording of sensor output.

\section{ACKNOWLEDGEMENT}

This work was sponsored by the Microsystems Technology Office of Defense Advanced Research Projects Agency (DARPA/MTO) and the Air Force Office of Scientific Research (USAF), under grant/contract number 49620-96-1-0376. The authors would like to thank Trevor Roper and Xuan-Qi Wang for their help with the process.

\section{REFERENCES}

[1] T.S.J. Lammerink, et al., "Micro-liquid flow sensor", Sensors and Actuators, A37-38, pp. 45-50, 1993.

[2] T.S.J. Lammerink, et al., "Modular Concept for Fluid Handling System", MEMS'96, pp. 389-394, February 1996, San Diego, California.

[3] M. Ashauer, et al., "Thermal Flow Sensor for Liquids and Gases, MEMS'98, pp. 351-355, January 1998, Heidelberg, Germany.

[4] M. Ritcher, M. Wackerle, P. Woias, B. Hillerich, "A novel flow sensor with high time resolution based on differential pressure principle," $M E M S$ '99, pp. 118123, January 1999, Orlando, FL.

[5] S. Wu, J. Mai, Y. Zohar, Y.C. Tai, and C.M. Ho, "A Suspended Microchannel with Integrated Temperature Sensors for High-pressure Flow Studies", MEMS'98, pp. 87-92, January 1998, Heidelberg, Germany.

[6] X.Q Wang, X. Yang, K. Walsh and Y.C. Tai, "Gas phase silicon etching with Bromine Triflouride," Transducer ' 97.

[7] G. Casella, R. L. Berger, Statistical Inference, pp. 216-228, 1990, Duxbury Press.

[8] F. M. White, Fluid Mechanics, $2^{\text {nd }}$ edition, McGraw-Hill, 1986. 